\title{
Reconfigurable Low Voltage Direct Current Charging Networks for Plug-in Electric Vehicles
}

\author{
Lesiba Mokgonyana, Kyle Smith and Stuart Galloway
}

\begin{abstract}
An emerging theme in the development of supporting facilities for plug-in electric vehicles (EVs) is the costeffective planning and utilisation of charging networks consistent with the uptake of EVs. This paper proposes a low voltage direct current (LVDC) reconfigurable charging network for plugin electric vehicles (EVs) and presents a functional energy management system (EMS) that is capable of planning and operating the charging network to minimise charging cost and to facilitate progressive infrastructure deployment based on EV demand. The charging network is connected to the main AC grid through one or more centralised AC/DC converters that supply a high power charge to EVs connected to the DC side of the converters. The EMS accommodates multiple parking bays, charging sources, AC constraints, non-linear EV battery loads and user charging requirements with a novel approach to managing user inconvenience. The inconvenience model is founded on the presence of user flexibility i.e., an allowance on charging time or battery SOC, providing the capability to increase asset utilisation and enable access for additional network users. Through a series of case studies and a stochastic forecasting approach, the reconfigurable network and EMS demonstrate the capacity to achieve savings over fixed AC and sequential DC systems.
\end{abstract}

Index Terms-Plug-in electric vehicles, direct current charging systems, mixed-integer program, charging infrastructure planning, multiple-charger control.

\section{INTRODUCTION}

$\mathbf{T}$ HE development of novel charging solutions for battery electric transportation systems (BETS) is an area of active research [1], [2], [3]. It is now widely accepted that electric vehicles (EVs), buses, commercial trucks, ships and potentially aircraft will become increasingly reliant upon battery electric power systems. The United Kingdom and France have banned the sale of fossil fuel vehicles from 2040 onwards [4] and, motivated by strict EURO7 emissions standards, major automotive manufacturers have announced new hybrid and full electric models to be brought into production from 2019-2022 [5]. The success of this electric transport revolution depends on several factors, one of which is the availability of battery charging infrastructure that can cost effectively integrate with the existing electrical network, deliver adequate energy transfer rates and adapt to the rapid technical development of this industry.

With respect to EVs, several research themes exist which seek to address the perceived and anticipated challenges that the widespread use of EVs may present. These challenges

The authors are with the Institute for Energy \& Environment, Department of Electronic and Electrical Engineering, University of Strathclyde, Glasgow G1 1XW, UK (e-mail: lesiba.mokgonyana@strath.ac.uk; kyle.smith@strath.ac.uk; stuart.galloway@strath.ac.uk). can be broadly classified as the impact of EV charging on the power network [6], [7], [8] ; the optimum deployment of charging infrastructure to minimise capital costs and maximise utilisation [9], [10], [11], [12]; and the correlation of EV charging with low carbon energy sources [13], [14].

Prior research has demonstrated that these challenges can be effectively addressed through the use of dedicated EV charging networks [15], [16]. The interconnection of the EV loads and point of common coupling (PCC) can be achieved using traditional AC power networks but there is a growing body of research that suggests low voltage direct current (LVDC) charging networks (400-1500 Vdc) can offer improved renewable energy integration by using fewer power conversion stages, enhanced network controllability due to the absence of reactive power and higher power charging compared to the existing AC charging solutions [17], [18], [19], [20]. However, the implementation of LVDC charging networks require the use of DC/DC converters at each parking bay to control the charging power flow and voltage for each vehicle. This adds an additional cost to enable charging for each parking bay and can introduce voltage stability challenges that must be addressed in the design and operation, furthermore, the centralized AC/DC converter and distribution cables are over sized to match the simultaneous demand from multiple vehicles. Such DC charging networks will operate well as high power chargers (HPC) in locations with frequent vehicle turnover such as dedicated charging stations in urban or motorway environments. But, under low utilization rates, the centralised converter will operate under part-load with lower power conversion efficiencies and the oversized, fixed cable, will result in higher implementation costs [21]. Although, with optimised LVDC charging infrastructure comes the issue of user inconvenience i.e. the ability of the network to reliably service the charging requirements of users as utilisation increases. The cost penalty frequently applied in other charging coordination problems to represent unmet charging requirements does not clearly capture user preference and flexibility therefore an alternative approach is proposed [2], [22], [23].

For longer duration EV charging scenarios such as work place, urban and residential overnight charging, this paper proposes a LVDC charging network that does not require DC/DC converters at each parking bay but instead takes advantage of the rapid start-up and shut-down properties of existing fast DC chargers [27] to reconfigure the charging network in a de-energized state. Power is routed to connected vehicles according to an optimised EMS solution. The EMS model constraints include single charger to EV pairings that are capable of varying power output over time, charging 
TABLE I: Plug-In EV Charging Networks

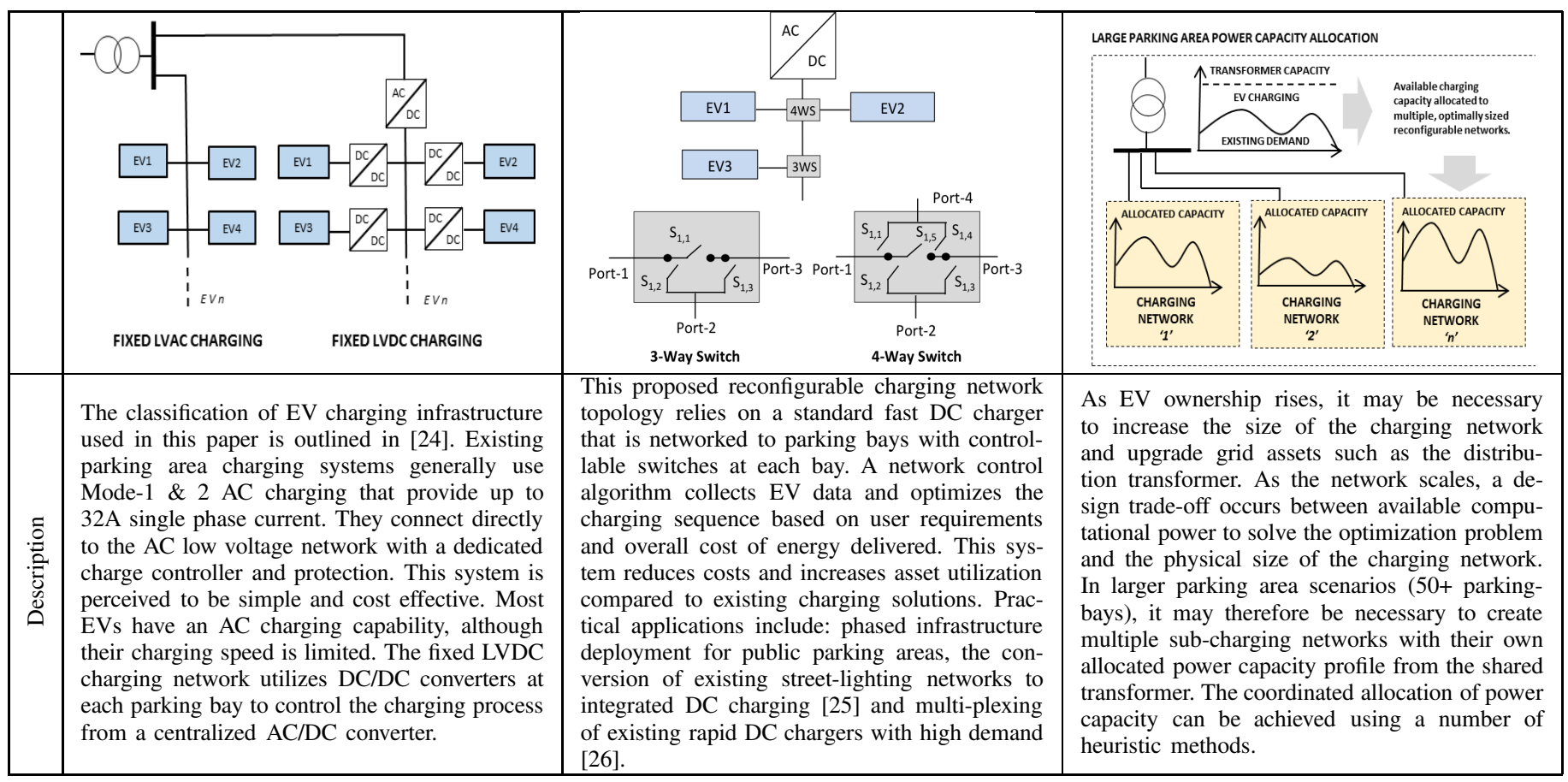

characteristics of lithium ion batteries and temporal transformer loading constraint. User inconvenience is defined and presented as a service selection matrix, enabling the user to choose between desired SOC level, parking time and cost to charge, which reflects the anticipated constraints on the charging network at any moment in time. This is formulated as a mixed integer linear programming (MILP) problem. The reconfigurable network is compared to the fixed DC charging networks discussed in prior literature and the $\mathrm{AC}$ charging systems currently being deployed. The solving speed and associated infrastructure costs for each solution are considered, which leads to a charging infrastructure deployment philosophy for long duration charging locations, that can be summarised as follows: 1) deploy minimal infrastructure (i.e. one charger and many plug-in points, controlled by this paper's proposed reconfigurable network model), 2) monitor utilisation of charger, 3) exploit user flexibility to maximise energy delivery based on this paper's proposed user inconvenience model (this approach allows the user to choose from several service options based on their own flexibility in advance of charging commencing), 4) deploy additional chargers when the level of user inconvenience is unacceptable, 5) upgrade public electrical network assets when charging demand is frequently curtailed.

Overall, this paper's proposal challenges the conventional, low-power AC charging systems that are deployed in parking areas for long duration charging by demonstrating the modularity, operation and economy of a reconfigurable LVDC charging network. The remainder of this paper is structured as follows. Section II provides an overview of the prominent EV charging configurations and the advantages that a reconfigurable DC charging network can offer. Section III presents the formulation of an EMS for the reconfigurable network with a representation of user charging inconvenience. Section IV introduces a series of case studies in which the control strategy and reconfigurable charging network are evaluated. Section V highlights the primary outcomes from this research and areas for further investigation.

\section{EV ChARGING NeTWORKS}

Table I provides a description of the standard AC charging network layout for parking areas and the fixed LVDC charging systems that has been discussed in prior literature [17], [18]. This paper proposes a reconfigurable DC charging network that can be considered a hybrid solution between the existing AC charging systems and the fixed LVDC charging network.

The reconfigurable aspect of this charging network circumvents some of the technical and standard limitations associated with DC distribution systems which are summarized in [28]. In this topology, fully depicted in Fig. 1, there is no requirement to interrupt DC current since the network is

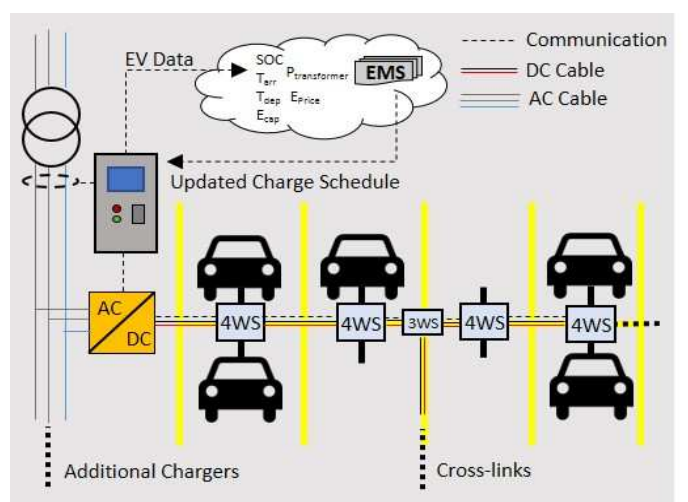

Fig. 1: Overview of a reconfigurable DC charging network. 
reconfigured offload and the instability challenges associated with constant power loads is mitigated as only one EV is charged from a dedicated source at any moment in time [29], [30]. Furthermore, in a reconfigurable network, the cables are sized for the maximum power of a single charger and not for the peak output power of multiple chargers, as is the case in a fixed LVDC charging network or standard AC network. A variety of existing switches and communication systems are commercially available which can be employed to operate the reconfigurable network. However, a failed switch could potentially block charging of other connected vehicles located beyond the point of failure unless specific provision is made to account for such a situation. Therefore, the central switch ( $S_{1,3}$ and $S_{1,5}$ in the reconfigurable diagram of Table I) should fail closed and the remaining switches open, to allow charging access to parking bays beyond the failed switch.

With existing EV charging schemes not extending beyond 100 parking bays at the upper end [31], this paper's reconfigurable DC charging network (covering up to 50 EV's) is suitable to current practical deployments but it could also be replicated many times over to accommodate future, larger parking areas without adversely affecting the solving speed of the EMS. In this case a zonal approach to charging is envisaged that would help manage the demand in line with the available capacity of secondary distribution transformers by allocating a portion of the available power capacity to each of the reconfigurable charging zones, as illustrated in Table I.

Regardless of the parking area size, to effectively operate a reconfigurable DC network (Fig. 1) requires an EMS solution that can optimally manage the interaction between the chargers and EVs according to user requirements and within specified system constraints. Next, we describe the mathematical model of the EMS, which performs network reconfiguration, charger power level selection and manages user inconvenience.

\section{Modeling \& Problem Formulation}

The practical benefits of the proposed reconfigurable LVDC charging network have been highlighted in Section II. However, the technical challenge relates to the EMS solution that must comply with the resultant constraints (e.g. EV and charger switching requirements). The following section introduces the problem formulation which takes the form of a MILP (or more specifically binary integer linear programming) problem. This optimization approach readily enables the consideration of discrete switching actions, which are essential to the operation of a reconfigurable network, and could not otherwise be modeled efficiently in linear programming or non-linear programming problems.

\section{A. Notation}

The notation defined below is employed for indices, sets, parameters and variables in the optimisation model.

$\begin{array}{ll}i & \text { Index for EV arrivals. } \\ k & \text { Index for EV chargers. } \\ \phi & \text { Index for charging power level. } \\ \Omega_{\mathrm{N}} & \text { Set of EV arrivals. } \\ \Omega_{\mathrm{L}} & \text { Set of charging power levels. }\end{array}$

\begin{tabular}{|c|c|}
\hline$\Omega_{\mathrm{M}}$ & Set of EV chargers. \\
\hline$\Omega_{\mathrm{T}}$ & Set of time intervals. \\
\hline$\eta_{i}$ & Charging efficiency. \\
\hline$E_{i}$ & Total energy supplied to $i$ th EV. \\
\hline$\Delta t$ & EMS charging time step. \\
\hline$t_{i}^{\mathrm{a}}$ & Time of arrival for $i$ th EV. \\
\hline$t_{i}^{\mathrm{d}}$ & Time of departure for $i$ th EV. \\
\hline$S_{i}^{i n i}$ & Initial $i$ th EV SOC (kWh). \\
\hline$S_{i}^{\text {fin }}$ & Final $i$ th EV SOC (kWh). \\
\hline$S_{i}^{1}$ & Start of constant voltage charging $(\mathrm{kWh})$. \\
\hline$S_{i}^{\ln }$ & Lower SOC level for SOC step $n$. \\
\hline$S_{i}^{u n}$ & Upper SOC level for SOC step $n$. \\
\hline$P_{k}^{r a t}(t)$ & Charging power rating according to EV SOC. \\
\hline$E^{c a p}$ & EV battery energy capacity $(\mathrm{kWh})$ \\
\hline$P_{i, k}^{\phi}$ & Charging power level. \\
\hline$\mu_{i}(t)$ & Availability status of $i$ th EV. \\
\hline$u_{i, k}^{\phi}(t)$ & $\begin{array}{l}\text { Binary variable denoting power level state } \\
\text { of charger output. }\end{array}$ \\
\hline$u_{i, k}(t)$ & $\begin{array}{l}\text { Binary variable representing control state for } i \text { th } \\
\mathrm{EV} \text { and } k \text { th charger over time interval } t \text {. }\end{array}$ \\
\hline$P_{i, k}(t)$ & $\begin{array}{l}\text { Power flow from } i \text { th } \mathrm{EV} \text { to } k \text { th charger over time } \\
\text { interval } t \text {. }\end{array}$ \\
\hline$S_{i}(t)$ & $\begin{array}{l}\text { SOC of } i \text { th accumulating over time } t \text { th interval } \\
(\mathrm{kWh}) \text {. }\end{array}$ \\
\hline$P_{n e t}^{\max }(t)$ & $\begin{array}{l}\text { Maximum power available from AC network } \\
\text { over time interval } t \text {. }\end{array}$ \\
\hline$C^{e}(t)$ & Cost of energy over time interval $t$. \\
\hline
\end{tabular}

\section{B. EV-Charger Switched Model}

The controller's task is to identify the optimum scheduling pattern between chargers and EVs that minimises the charging costs according to the system constraints. The following sections introduce the modeling approach, which has been structured as a MILP problem that can be solved using the branch-and-bound method within Matlab or other commercial solvers [32]. The MILP problem may be applied as either a network planning tool — to determine the minimum charging infrastructure requirements that will satisfy EV user demands — and as a near real-time energy management system. The EMS operates in near real time in the sense that each schedule update is completed within the stated time step.

The proposed model is formulated to determine the optimal switching sequence for minimizing the total energy cost. Let $u_{i, k}(t) \in[0,1]$ denote the binary control state for $i$ th EV and $k$ th charger pair over time interval $t$. The objective function is expressed as;

Problem P1 (Whole Optimisation Model),

$$
J=\min _{u_{i, k}(t)} \sum_{t \in \Omega_{\mathrm{T}}} \sum_{i \in \Omega_{\mathrm{N}}} \sum_{k \in \Omega_{\mathrm{M}}} P_{i, k}(t) u_{i, k}(t) C^{\mathrm{e}}(t) \Delta t,
$$

subject to

1) Exclusive EV charging: A charger is only allowed to charge one EV at a time,

$$
\sum_{i \in \Omega_{\mathrm{N}}} u_{i, k}(t) \leq 1, \forall k, \forall t
$$


and an EV must only receive power from single charger at a time,

$$
\sum_{k \in \Omega_{\mathrm{M}}} u_{i, k}(t) \leq 1, \forall i, \forall t .
$$

2) Temporal Grid Capacity: Over each time interval, the total power consumed by the charging network must not exceed available grid capacity,

$$
\sum_{i \in \Omega_{\mathrm{N}}} \sum_{k \in \Omega_{\mathrm{M}}} P_{i, k}(t) u_{i, k}(t) \leq P_{\mathrm{net}}^{\max }(t), \forall t .
$$

3) Energy Requirement: The energy supplied over the charging period must be equal to the energy required by the EV.

$$
\begin{gathered}
\eta_{i} \sum_{t \in \Omega_{\mathrm{T}}} \sum_{k \in \Omega_{\mathrm{M}}} P_{i, k}(t) u_{i, k}(t) \Delta t=E_{i}, \forall i, \\
E_{i}=\left(\mathrm{S}_{i}^{\mathrm{fin}}-\mathrm{S}_{i}^{\mathrm{ini}}\right) .
\end{gathered}
$$

The SOC of each EV accumulates over time,

$$
S_{i}(t)=S_{i}(0)+\eta_{i} \sum_{\tau \in \Omega_{t}} \sum_{k \in \Omega_{\mathrm{M}}} P_{i, k}(\tau) u_{i, k}(\tau) \Delta t, \forall i,
$$

where $\Omega_{t}=[0, t)$.

$$
S_{i}^{\min } \leq S_{i}(t) \leq S_{i}^{\max }, \forall i, \forall t .
$$

The formulation, (1)-(8), represents EV-charger switching control in terms of linear functions of variable $u_{i, k}(t)$, forming a MILP problem. Next, a dynamic model to control power output and allocate charging intervals is introduced within the same MILP structure. However, if the power $P_{i, k}(t)$ varies then the functions become non-linear. Next, a dynamic model to control power output and allocate charging intervals is introduced within the MILP structure.

\section{Power Control and Interval Allocation}

The charging system must be able to determine appropriate power outputs given a multitude of network configurations and charging requirements. EV-charger switch control and power level states are linked as follows:

$$
\begin{gathered}
u_{i, k}(t)=\sum_{\phi \in \Omega_{\mathrm{L}}} u_{i, k}^{\phi}(t) \mu_{i}(t), \\
\sum_{\phi \in \Omega_{\mathrm{L}}} u_{i, k}^{\phi}(t)=1,
\end{gathered}
$$

each EV is available to charge during the interval between its times of arrival and departure,

$$
\mu_{i}(t)= \begin{cases}1, & t_{i}^{\mathrm{a}}<t<t_{i}^{\mathrm{d}} \\ 0, & \text { otherwise }\end{cases}
$$

For every $u_{i, k}^{\phi}(t)$, there is a corresponding power level, $P_{i, k}^{\phi}$. Therefore,

$$
P_{i, k}(t) u_{i, k}(t)=\sum_{\phi \in \Omega_{\mathrm{L}}} P_{i, k}^{\phi} u_{i, k}^{\phi}(t) \mu_{i}(t) .
$$

The charging profile for lithium ion batteries is adapted from typical characteristics to make it suitable for the MILP

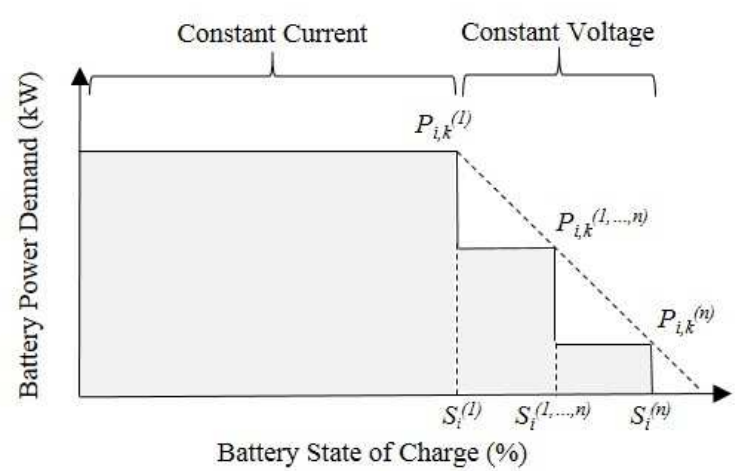

Fig. 2: Stair-step charging profile approximation.

formulation [11], [33]. The typical and stair-step charging profiles are shown in Fig. 2.

$$
\begin{gathered}
P_{i, k}(t)= \begin{cases}P_{k}^{\text {rat }}, & \mathrm{S}_{i}^{\text {fin }} \geq \mathrm{S}_{i}(t) ; \\
\frac{P_{k}^{\text {rat }}\left(100-\mathrm{S}_{i}(t) / /^{\text {cap }}\right)}{\left(100-\mathrm{S}_{i}^{1} / E^{\text {cap }}\right)}, & \text { otherwise. }\end{cases} \\
S_{i}^{(l n)} \leq S_{i}(t) \leq S_{i}^{(\text {un })},
\end{gathered}
$$

where $P_{i, k}(t) \leq P_{i, k}^{1}$ if $S_{i}^{(l 1)} \leq S_{i}(t) \leq S_{i}^{(u 1)}$, $P_{i, k}(t) \leq P_{i, k}^{2}$ if $S_{i}^{(l 2)} \leq S_{i}(t) \leq S_{i}^{(u 2)}$ and so forth.

In summary, the problem formulation, (1)-(14), describes a MILP model to optimise switching links between EVs and chargers with varying power supply. The optimisation model is perfectly suited to reconfigurable networks such as the network illustrated in Fig. 1. However, given some user inputs or congested networks a feasible solution may not exist. In this case, the output of the optimisation routine will be "infeasible", even though it may be possible to amend the user's requirements. The following section extends the above model to effectively characterise flexible users.

\section{Managing User Inconvenience}

There will be occasions when the charging network cannot deliver the preferred charging service for the joining EV user due to a congested charging schedule or constraints on the $\mathrm{AC}$ distribution network. This represents a user inconvenience scenario as the user cannot receive their desired SOC level within their parking time. Previous EV charging coordination papers propose a cost penalty to the CPO for failing to meet the user's desired SOC before the user departs the charging network [11], [9]. This penalty approach enables the EMS to identify the least cost charging schedule for the group of EVs by inconveniencing some users and penalizing the CPO for doing so. However, in a practical charging context, this paper's proposed EMS can provide the user with an upfront charging service selection that guarantees a specific service in advance of charging. The flow chart in Fig. 3 demonstrates that a selection matrix is composed of $m$ SOC levels and $n$ parking times. The EMS generates $m \times n$ optimisation results based on the joining EV parameters and a 'rolling-schedule' for the network which includes the temporal transformer loading and 
utilisation of each charger. Each instance within the rollingschedule relies on the problem stated mathematically in Problem P2. In the formulation, the notation $x^{\prime}$ denotes variables for the new EV arrival. Note that the dimension of Problem P2 is smaller than that of the whole optimisation model (Problem P1) because it determines the potential charging schedule for the latest EV to join the network rather than the entire network simultaneously.

Problem P2 (User Inconvenience Model).

$$
\min _{u_{k}^{\prime}(t)} \sum_{t \in \Omega_{\mathrm{T}}} \sum_{k \in \Omega_{\mathrm{M}}} P_{k}^{\prime}(t) u_{k}^{\prime}(t) C^{\mathrm{e}}(t) \Delta t+J,
$$

subject to

$$
\begin{gathered}
u_{k}^{\prime}(t) \leq 1-\sum_{i \in \Omega_{\mathrm{N}}} u_{i, k}(t), \forall k, \forall t \\
\sum_{k \in \Omega_{\mathrm{M}}} u_{k}^{\prime}(t) \leq 1-\sum_{k \in \Omega_{\mathrm{M}}} u_{i, k}(t), \forall i, \forall t \\
\sum_{k \in \Omega_{\mathrm{M}}} P_{k}^{\prime}(t) u_{k}^{\prime}(t) \leq P_{\mathrm{net}}^{\max }(t)-\sum_{i \in \Omega_{\mathrm{N}}} \sum_{k \in \Omega_{\mathrm{M}}} P_{i, k}(t) u_{i, k}(t), \forall t \\
\eta^{\prime} \sum_{t \in \Omega_{\mathrm{T}}} \sum_{k \in \Omega_{\mathrm{M}}} P_{k}^{\prime}(t) u_{k}^{\prime}(t) \Delta t=E^{\prime} \\
S^{\prime}(t)=S^{\prime}(0)+\eta^{\prime} \sum_{\tau \in \Omega_{t}} \sum_{k \in \Omega_{\mathrm{M}}} P_{k}^{\prime}(\tau) u_{k}^{\prime}(\tau) \Delta t
\end{gathered}
$$

The rolling-schedule is updated every time step with an optimisation of the whole system (Problem P1), which takes into consideration both the newly arrived EVs and any EVs that have departed before their scheduled charge has completed. However, as the utilization of the charging network increases, with the diffusion of EV ownership, there will be occasions when even the user selection matrix cannot offer all services due to congestion caused by a limited number of centralized DC chargers or a power constraint on the secondary distribution transformer. If this scenario arises frequently, it is an indication to the charging network operator that it is time to install an additional DC charger or to issue a request to the Distribution Network Operator to increase transformer capacity.

\section{E. Economic Analysis}

The feasibility of a reconfigurable DC charging network is compared to the standard AC charging systems that are currently being installed for long-duration charging solutions. This analysis requires an assessment of net present value (NPV) for each investment:

$$
N P V(j, N)=\sum_{y=0}^{N} \frac{R_{y}}{(1+j)^{y}} .
$$

As outlined in (21), the NPV is composed of: the net annual revenue which is the product of the charging price and energy delivered to EVs minus the annual cost of energy to service the EV charging demand $\left(R_{y}\right)$; the investment discount rate $(j)$; the time period in which revenue is generated $(y)$; and the total number of periods in which the investment is evaluated

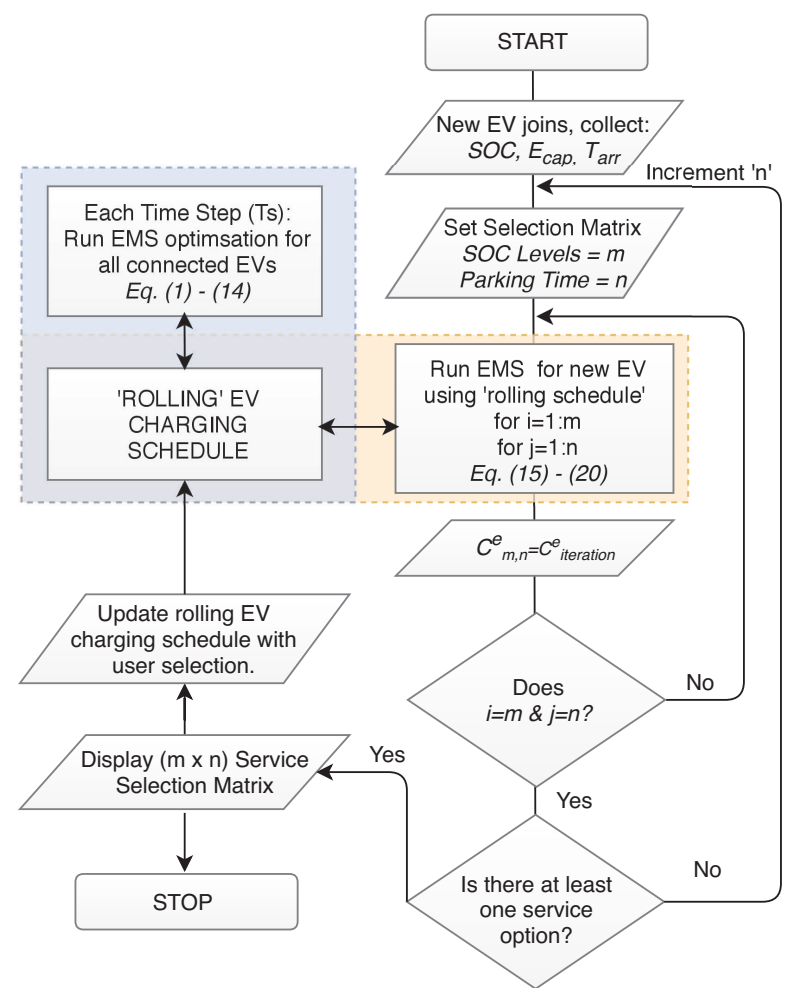

Fig. 3: Generation of the rolling-schedule and service selection matrix to manage user inconvenience.

$(N)$. This analysis does not take into account annual servicing and administration costs or installation costs, it is assumed that these costs will be similar in both cases.

The economic model and preceding optimisation model are applied to the planning and operation of EV charging networks.

\section{Case Studies \& Simulations}

The performance of the developed EMS is assessed as both a network-planning tool and as a near real time network controller. In both applications, three practical deployment environments are simulated in which a reconfigurable LVDC charging network may prove beneficial. These include work place parking, urban parking lots and residential overnight parking. These cases were identified as the most appropriate charging locations in an expansive study of electric vehicle ownership and user habits in the United States [34].

\section{A. EV Charging Network Parameters}

To test the EMS it is necessary to develop a set of input parameters that simulate the expected arrival/departure times of vehicles at each location and the associated SOC for each $\mathrm{EV}$, these are outlined in Table II based on similar approaches from [1] and [33]. In addition, realistic secondary distribution transformer loading profiles and TOU pricing are required to simulate real-life constraints on the charging network. In each charging location, a set of $50 \mathrm{EV}$ charging parameters are generated, as depicted in Fig. 4. From this set of 50 parameters, 

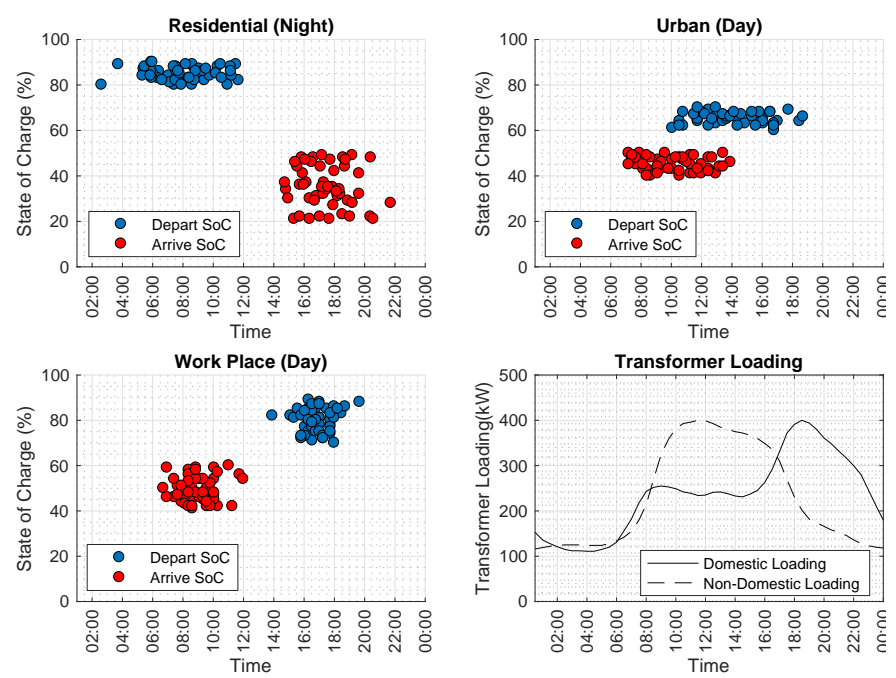

Fig. 4: Arrival time, departure time and state of charge probability distributions for each case study location.

each simulation randomly selects a subset of EVs as the input parameters for the EMS.

In practical deployment scenarios, it is likely that the charging network will connect to an existing secondary distribution transformer and therefore the EMS must be sympathetic to the existing loading conditions on said transformer. Two loading profiles from Elexon's demand classification system are utilised [35] (Elexon is the organisation that manages Great Britain's transmission system balancing). The winter demand profile for a Domestic Class-1 profile is used for the Residential charging scenario and the winter demand profile for a Non-domestic Class-3 profile is applied to the Urban and Work Place charging scenarios. In both cases the winter profile is selected to simulate the worst case loading condition and in each charging scenario, the loading profiles are scaled to suit a 500kVA distribution transformer capacity.

It is unknown whether commercial EV charging infrastructure operators will possess the ability to access wholesale electricity prices perhaps this will occur when an operator

TABLE II: Arrival \& Departure Probability Distributions

\begin{tabular}{lll}
\hline Location & Arrival/Departure Times & Arrival/Departure SOC \\
\hline Work Place & Normally distributed & Uniform distribution with \\
& around 09:00 for arriving & an arrival SOC between \\
& EVs and normally & $30-40 \%$ and a departure \\
& distributed around 17:00 & SOC between 70-80\%. \\
& for departing EVs, both & \\
& with a 1 hour variance. & \\
Residential & Normally distributed & Uniform distribution with \\
& around 17:00 for arriving & an arrival SOC between \\
& EVs and normally & 20-50\% and a departure \\
& distributed around 08:00 & SOC between 85-100\%. \\
& for departing EVs, both & \\
& with a 1 hour variance. & \\
& Uniformly distributed be- & Uniform distribution with \\
& tween 09:00 to 17:00. & an arrival SOC between \\
Urban & & $40-50 \%$ and a departure \\
& & SOC between 60-70\%. \\
\hline
\end{tabular}

reaches a certain scale. In the meantime, it is likely the operator will be subject to standard energy supplier tariffs, either flat-rate or TOU prices. In the UK, TOU tariffs are not commonly used, however, the closest available tariff is the Economy 10 tariff offered by SSE [36]. The SSE Economy 10 tariff is available in many regions throughout the UK but prices vary according to location. This tariff provides 10 hours of off-peak energy pricing during the day at a rate of $£ 0.1162 / \mathrm{kWh}$ and 14 hours of peak pricing at $£ 0.1979 / \mathrm{kWh}$ for the distribution zone located around the city of Glasgow, Scotland [36]. The Economy 10 tariff is applied as the energy pricing parameter for all charging scenarios, this is compared against a standard flat-rate tariff of $£ 0.164 / \mathrm{kWh}$ that is offered in the same geographic region.

\section{B. Case Study 1: EMS Validation}

Fig. 5 demonstrates the charging schedule for five EVs connected to a charging network with two, 50kW rapid DC chargers and using test EV input parameters from Table III. It is clear from the characteristics for EV-2, that Charger1 and Charger-2 operate independently and only one EV is charged from each charger at any moment in time. In this test scenario, and in further investigations, the EMS can select one of three different charging power levels $(10 \mathrm{~kW}, 30 \mathrm{~kW}$ and $50 \mathrm{~kW})$. These power levels enable a scaled reduction in power when the EV battery surpasses its constant current charging threshold, however, these variable power levels can also be utilized during the charging routine to meet the user requirements at the least cost and within the available power capacity of the transformer (Fig. 6). Additional power levels can be incorporated to offer increased power control but this must be balanced against the increased computational complexity that would result.

The computational speed to convergence of the EMS optimisation process is influenced by the time-step size selection. In the five EV, two charger test network a time step of 30 mins is adopted. This is an appropriate starting point considering the energy market balancing and settlement process is conducted in half-hourly periods [35]. However, a smaller time step will increase the solving time but improve the accuracy of the charging cost. Table IV demonstrates the effect of varying time steps on the optimization solving speed and the cost to charge a representative sample of $25 \mathrm{EVs}$ and $50 \mathrm{EVs}$ with four chargers and five chargers respectively. To achieve reasonable solving speeds for the $50 \mathrm{EV}$ network, it is necessary to limit the time horizon to the most relevant time periods rather than consider an entire 24-hour period. In all scenarios the EMS solves within the allocated time-step. In these simulations and subsequent scenarios, Matlab is used to perform the EMS optimization process on a $3.4 \mathrm{GHz}$ Intel Core i7 processor.

The solving times and network scale are appropriate for the desired applications - long duration charging $(>15$ minute parking time) and the integration of charging infrastructure into existing electrical network infrastructure with minimal initial upgrade requirements. Larger parking areas may contain multiple separately controlled reconfigurable networks or one continuous network that is controlled by a more powerful 


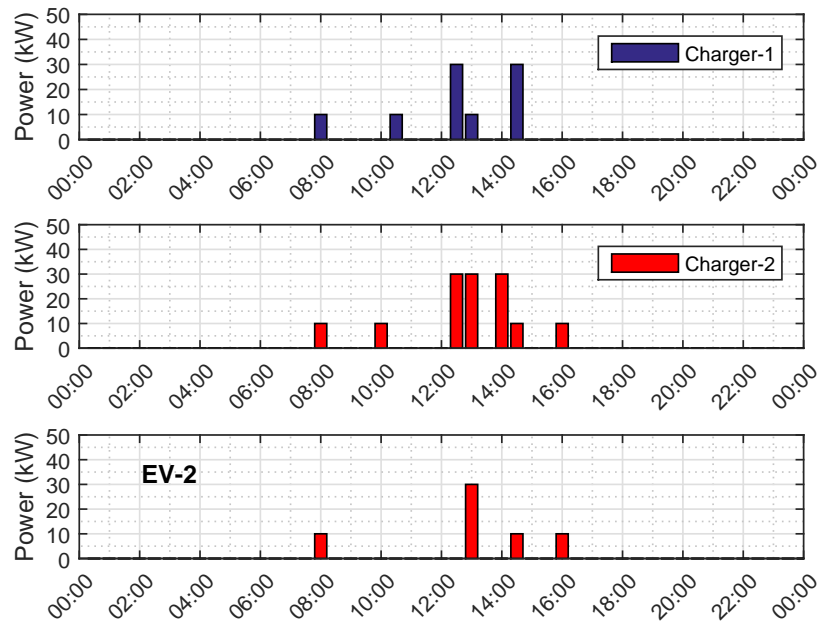

Fig. 5: Overall charging characteristics for the five-EV, twocharger system and an individual characteristic for EV-2.

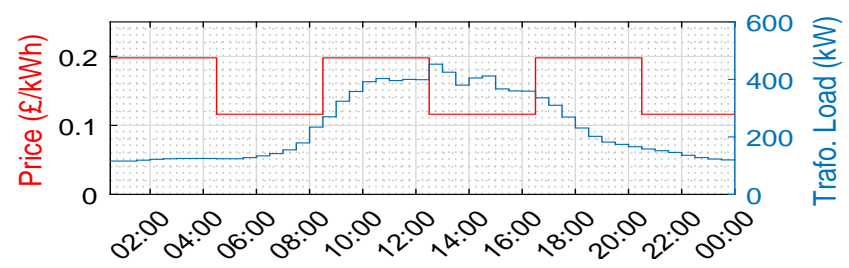

Fig. 6: TOU pricing and transformer load.

cloud based processor, as depicted in Fig. 1. The size of each charging network is dictated by the physical limitations of cable runs, allowable voltage drops and acceptable computational complexity.

\section{Case Study 2: Charging Infrastructure Performance}

This study demonstrates the EMS as an infrastructure planning tool and highlights the value of an optimised reconfigurable DC network compared to a reconfigurable DC network that charges vehicles sequentially upon arrival, and the existing AC charging networks that charge EVs immediately upon connection to the network. The parameters used in this analysis are presented in Table $\mathrm{V}$. The charging price is set to $£ 0.25 / \mathrm{kWh}$ which is similar to pricing offered by commercial operators [37]. The $50 \mathrm{~kW}$ DC and $7 \mathrm{~kW}$ AC chargers are priced according to [38] and [39]. The cable cross sectional area (CSA) was calculated according to BS7671 standards. The DC cable has 3-cores and the AC cable 4-cores [40], both cables are copper conductors, XLPE insulated and Steel

TABLE III: Input Parameters for $5 \mathrm{EV} \times 2$ Charger Network

\begin{tabular}{ccccc}
\hline $\mathrm{EV}_{\mathrm{n}}$ & $\mathrm{kWh}_{\text {start }}$ & $\mathrm{kWh}_{\text {end }}$ & $\mathrm{T}_{\text {arrival }}$ & $\mathrm{T}_{\text {depart }}$ \\
\hline $\mathrm{EV}_{1}$ & 20 & 50 & $10: 00$ & $15: 00$ \\
$\mathrm{EV}_{2}$ & 30 & 60 & $08: 00$ & $17: 00$ \\
$\mathrm{EV}_{3}$ & 40 & 50 & $09: 00$ & $11: 00$ \\
$\mathrm{EV}_{4}$ & 20 & 40 & $07: 00$ & $15: 00$ \\
$\mathrm{EV}_{5}$ & 20 & 40 & $05: 00$ & $13: 00$ \\
\hline
\end{tabular}

TABLE IV: Solving Time Comparisons: $4 \mathrm{CH}$ x $25 \mathrm{EV}$ and $5 \mathrm{CH} \times 50 \mathrm{EV}$.

\begin{tabular}{cccccccc}
\hline & \multicolumn{3}{c}{ Work Place } & \multicolumn{2}{c}{ Urban } & \multicolumn{2}{c}{ Residential } \\
\hline Network & $\Delta t$ & Time $(\mathrm{s})$ & $\operatorname{Cost}(£)$ & Time $(\mathrm{s})$ & $\operatorname{Cost}(£)$ & Time $(\mathrm{s})$ & $\operatorname{Cost}(£)$ \\
\hline \multirow{3}{*}{$4 \mathrm{CHx} 25 \mathrm{EV}$} & 15 & 151 & $£ 51.71$ & 142 & $£ 34.13$ & 170 & $£ 83.29$ \\
& 30 & 28 & $£ 54.16$ & 12 & $£ 36.15$ & 21 & $£ 83.49$ \\
& 60 & 4 & $£ 68.28$ & 5 & $£ 43.53$ & 5 & $£ 86.24$ \\
$5 \mathrm{CHx} 50 \mathrm{EV}$ & 15 & 321 & $£ 127.54$ & 10 & $£ 78.33$ & 537 & $£ 211.94$ \\
& 30 & 31 & $£ 129.00$ & 7 & $£ 78.12$ & 396 & $£ 190.87$ \\
& 60 & 9 & $£ 127.58$ & 1 & $£ 88.16$ & 116 & $£ 177.13$ \\
\hline
\end{tabular}

TABLE V: Simulation Parameters for Case Study 2

\begin{tabular}{cc}
\hline Parameter & Value \\
\hline Number of EVs & 50 \\
EV Battery Capacity & $60 \mathrm{kWh}$ \\
Power Rating DC Charger & $50 \mathrm{~kW}$ \\
Number of DC Chargers & 5 \\
Power Rating of AC Chargers & $7 \mathrm{~kW}$ \\
Charger $\eta$ for both AC \& DC & $100 \%$ \\
Simulation Time Step & 30 minutes \\
Simulation Time Period & 24 hours \\
Power Level 1: 0-90\% SOC & $50 \mathrm{~kW}$ \\
Power Level 2: 90-95\% SOC & $30 \mathrm{~kW}$ \\
Power Level 3: 95-100\% SOC & $10 \mathrm{~kW}$ \\
Assumed Investment Period & 10 years \\
Discount Rate & $5 \%$ \\
Charging Price & $£ 0.25 / \mathrm{kWh}$ \\
\hline
\end{tabular}

Wire Armored (SWA). It was assumed that all parking bays are within $100 \mathrm{~m}$ of a centralized $50 \mathrm{~kW}$ DC charger with a maximum acceptable voltage drop of $3 \%$.

In the first instance, it is desirable to compare the performance of the LVDC reconfigurable charging network against established charging solutions in order to quantify the benefit it brings. The baseline charging network (AC Uncontrolled) uses a standard $7 \mathrm{~kW}$ AC charger supplied to each parking-bay, this represents the existing charging infrastructure planning theory for extended-stay parking areas. This is compared to a reconfigurable DC charging network (DC Uncontrolled) that charges EV's in sequential order (first come, first served). Both methods are compared against the proposed EMS controller (DC Controlled) presented in Section III. The simulations are performed in three charging location scenarios: work place, residential and urban areas. The resulting charging demand profiles on the secondary distribution transformer are displayed in Fig. 7. In each charging scenario the $7 \mathrm{~kW}$ AC charging network and 50kW DC sequential charging system either approach or surpass the $500 \mathrm{kVA}$ capacity of the distribution transformer, whereas the optimised EMS ensures the charging schedule for the reconfigurable DC network remains within the power limit constraint of the transformer and maximizes energy delivery during the off-peak pricing periods for every scenario. Table VI highlights the charging costs associated with servicing $50 \mathrm{EVs}$ at each location. As expected, the optimized EMS charging schedule has the least expensive charging cost in all scenarios.

Table VII considers the cost implications of reducing the number of chargers from five, $50 \mathrm{~kW}$ chargers, to four and increasing to six chargers. It is clear that increasing the number of chargers has no affect on the daily cost of energy but reducing the number of chargers to four marginally increases 


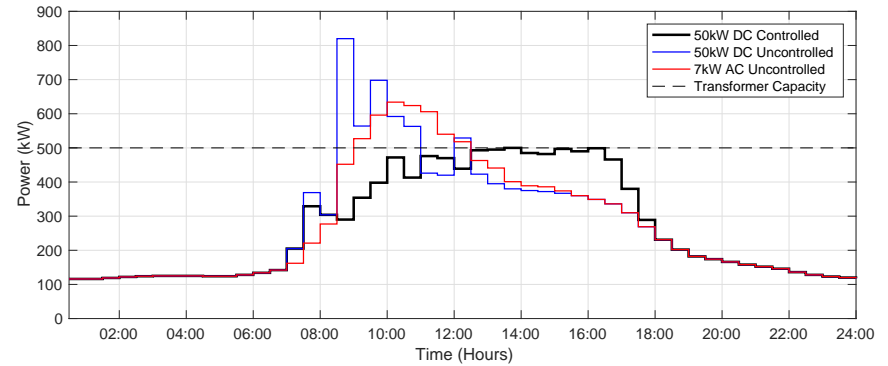

(a) Work Place charging profiles for $50 \mathrm{EVs}$.

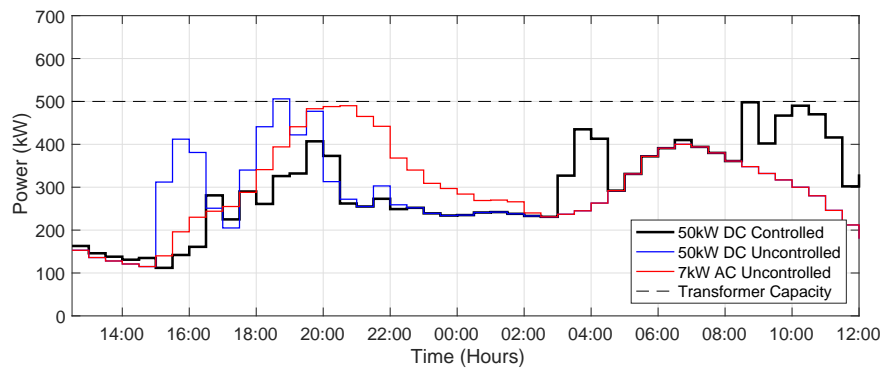

(b) Residential charging profiles for 50 EVs.

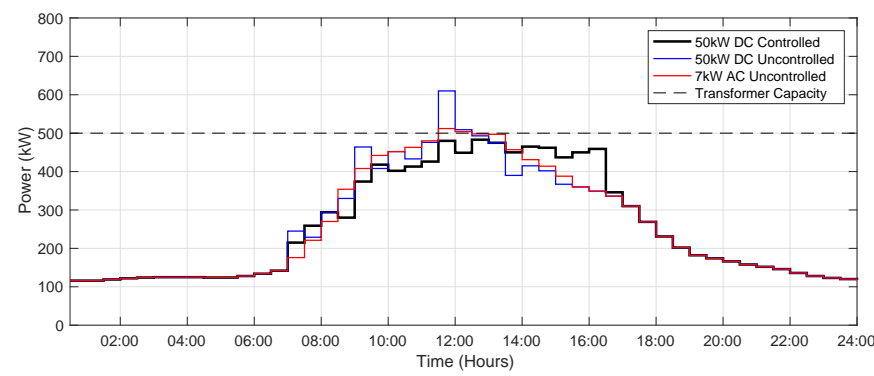

(c) Urban charging profiles for 50 EVs.

Fig. 7: Transformer loading for $50 \mathrm{EVs}$ and different charging coordination methods.

TABLE VI: Service Cost Comparison of Charging Scenarios for 5 Chargers \& $50 \mathrm{EVs}$

\begin{tabular}{ccccc}
\hline Location & $\begin{array}{c}50 \mathrm{~kW} \text { DC } \\
\text { Optimized }\end{array}$ & $\begin{array}{c}50 \mathrm{~kW} \text { DC } \\
\text { Sequential }\end{array}$ & $7 \mathrm{~kW} \mathrm{AC}$ & Flat Tariff \\
\hline Urban & $£ 86.10$ & $£ 185.46$ & $£ 99.48$ & $£ 102.50$ \\
Work Place & $£ 128.94$ & $£ 188.72$ & $£ 172.93$ & $£ 152.52$ \\
Residential & $£ 182.06$ & $£ 182.70$ & $£ 246.78$ & $£ 246.00$ \\
\hline
\end{tabular}

the daily cost of energy. Despite the daily cost increase the NPV of the charging infrastructure over a 10 year period with a 5\% discount rate is significantly higher for a four charger system, compared to a five charger system and higher still compared to the normal $\mathrm{AC}$ charging infrastructure that is currently deployed, indicating a superior investment opportunity. It should be mentioned that the annual revenue remains constant across all scenarios as this is an infrastructure assessment analysis that assumes the same forecasted number of EVs, arrival rates and charging requirements across all four scenarios. The EMS optimisation approach is applied as a planning tool to determine the minimum number of chargers necessary to meet the forecasted EV charging demand.
TABLE VII: Infrastructure Assessment for $50 \mathrm{EVs}$

\begin{tabular}{lllll}
\hline Design Parameter & $4 \times 50 \mathrm{~kW}$ & $5 \times 50 \mathrm{~kW}$ & $6 \times 50 \mathrm{~kW}$ & $50 \times 7 \mathrm{~kW}$ \\
& DC & DC & DC & AC \\
\hline Daily Energy Cost & $£ 129.43$ & $£ 128.94$ & $£ 128.94$ & $£ 172.93$ \\
Annual Energy Cost & $£ 32,357$ & $£ 32,235$ & $£ 32,235$ & $£ 43,232$ \\
Charger Cost & $£ 80,000$ & $£ 100,000$ & $£ 120,000$ & $£ 17,850$ \\
Peak AC Power & $200 \mathrm{~kW}$ & $250 \mathrm{~kW}$ & $300 \mathrm{~kW}$ & $350 \mathrm{~kW}$ \\
Cable Capacity & $50 \mathrm{~kW}$ & $50 \mathrm{~kW}$ & $50 \mathrm{~kW}$ & $350 \mathrm{~kW}$ \\
Cable CSA & $50 \mathrm{~mm}^{2}$ & $50 \mathrm{~mm}^{2}$ & $50 \mathrm{~mm}^{2}$ & $240 \mathrm{~mm}^{2}$ \\
Cable Cost & $£ 916$ & $£ 916$ & $£ 916$ & $£ 6,180$ \\
Annual Income $(£ 0.25 / \mathrm{kWh})$ & $£ 58,125$ & $£ 58,125$ & $£ 58,125$ & $£ 58,125$ \\
\hline 10 Year NPV & $£ 112,436$ & $£ 94,285$ & $£ 75,238$ & $£ 86,752$ \\
\hline
\end{tabular}

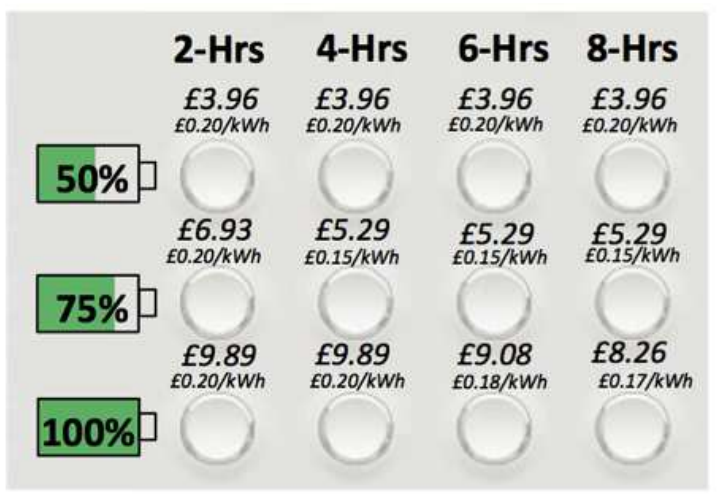

Fig. 8: EV charging service selection matrix

\section{Case Study 3: Service Selection Matrix}

The EMS can equally be applied to the near real time energy management of the charging network. The reconfigurable DC charging network is designed to maximize the utilization of the fixed infrastructure and as a result the network will naturally become constrained as EV utilization increases due to limitations on charger power output capacity, available headroom on the distribution transformer and volatility in energy prices. It is therefore necessary to offer the EV user a selection of charging services that take into consideration the existing charging schedule and future constraints. This permits the user to select the most appropriate departure SOC, departure time and price for the service. In line with existing pay in advance parking system arrangements in the UK - the driver selects and pays for a fixed period of time on arrival in the parking area and no compensation is available if they vacate before the prepaid period elapses. The EMS and selection matrix presented in this paper follows this established practice and therefore, if a user returns prior to the agreed charging completion time, there will be no cost re-adjustment, however, the additional charging capacity made available by the early-to-depart EV will be incorporated in the next optimization step.

To produce these charging service selections, the online EMS algorithm must generate multiple service options based on the EV's SOC upon joining the network, the EV battery capacity and the existing network charging schedule. From a practical perspective, the user selection matrix could be generated between the time the user plugs into the network and walks over to a centralised payment kiosk or the user may be able to wait longer, perhaps for a charging notification 
and payment to appear on the user's mobile device. The EMS should therefore be able to provide an immediate service option while continuing to improve the accuracy and expand the user options, prior to the user making a selection.

A user-selection matrix from simulated results is presented in Fig. 8 that offers the EV owner three different SOC levels and four different parking time options. The costs for each charging service option represent the direct energy cost to the CPO. To generate the service selection matrix requires twelve, independent optimization runs with discrete combinations of SOC and parking time. The results in Fig. 8 are from two $50 \mathrm{~kW}$ DC chargers networked to twenty-six EV parking bays at a work place parking area. In this scenario, the selection matrix is the result of a whole network optimization and demonstrates that the network can accommodate all service options for the 26th EV, however, from Table IV it is clear that this matrix generation approach takes over five minutes to produce using 30 minute time steps. From a practical implementation perspective, the rolling-schedule optimization can generate the service selection matrix in less than 5 seconds. Although this approach is more practical in solving speed, it can result in fewer available service options than the slower, whole network optimization. In this congested network scenario the 'rolling-schedule' can only accommodate the 26th $\mathrm{EV}$ if it remains parked for at least 9 hours. However, if the user can wait an extra 50 seconds prior to making their service selection, the EMS continues to refine the service options by generating a service selection matrix based on the 60 minute time step as outlined in Table IV. Then once complete, and if time permits, the EMS can move onto the 30 minute time step with the resulting matrix in Fig. 8. This tiered approach to the selection matrix balances the operational need for rapid service options against the optimal network solution and computational time constraints.

The selection matrix scenario in Fig. 8 represents the service costs for a twenty-sixth EV to join the network at 9am and with a starting SOC of $10 \mathrm{kWh}$. As expected, the overall service cost for each SOC level reduces as the parking time increases. However, the per unit tariff rate varies widely across the service options therefore both the unit rate of energy and the total service cost should be provided so that the user may choose their preferred option. The best economic choice for the EV user in this scenario is a 4-hour parking time with a $75 \%$ SOC on completion. The 50\% SOC rates appear higher because the highest power level is not being used as it would supply more energy than required (this charging service option requires $20 \mathrm{kWh}$ but a $50 \mathrm{~kW}$ power level would deliver $25 \mathrm{kWh}$ during the allocated 30 minute time-step). Thus less energy is supplied during off-peak periods for this service option as the lower power levels $30 \mathrm{~kW}$ and $10 \mathrm{~kW}$ must be used according to the model's SOC and power level constraints, (12)-(14). Using a lower time step, e.g. 15 minutes, will improve the results but another factor plays a key role: although the use of the 30-minute time step makes the results suboptimal, it is more computationally efficient as explained in Section IV-B. Despite the sub-optimal solution, the proposed charging system still delivers a lower daily charging cost and NPV in comparison to a first come, first served charging pattern for conventional
AC charging systems. It is also important to note that the EMS is minimizing the cost for the charging network operator and not the price for the connecting EV, therefore each of the available service options are presented in the context of independent charging schedules. This means the 26th EV must pay the difference between the previously agreed charging cost for the original $25 \mathrm{EVs}$ and one of the new charging network cost options based on the newly optimised charging schedules for $26 \mathrm{EVs}$ in the user selection matrix.

It is intuitive to offer a reduced charging cost to an EV user that is connected to the network for a longer duration, as this allows the EMS to charge the vehicle at the lowest prices and schedule it around more time urgent EV users. However, this is in contrast with conventional parking-lot pricing strategies where a vehicle owner will pay according to the time spent occupying a parking-bay. It is perhaps then important to state that this service selection matrix only presents the cost of energy and assumes there is no additional charge to park an $\mathrm{EV}$ for longer durations.

\section{CONCLUSIONS}

A reconfigurable LVDC charging network for plug-in electric vehicles along with an EMS controller are proposed. The results of the proposed model have interesting implications for charging network design and operation. Specifically, despite the use of fast DC chargers being more capital intensive than conventional $\mathrm{AC}$ equivalents, and their adoption to date being limited, this should not be considered a deterrent to their use because they can have comparable or even lower overall costs in the long term. For this to be realised, a network of DC chargers must be deployed optimally, with three main factors influencing design and operation costs. These are the total number of chargers, network configurability and energy management. As demand grows, operators should consider taking advantage of user flexibility before upgrading their networks, while further work could demonstrate the value of incorporating stationary battery storage with local renewable energy resources. The inconvenience technique within the proposed model enables operators to create charging offers that can be easily interpreted by those with flexible time or SOC requirements and are tailored to the current network utilisation level. This is a mutually beneficial approach, providing additional revenue for operators and access for new EV arrivals in congested charging networks.

\section{ACKNOWLEDGEMENT}

This work has been supported through the EPSRC Centre for Doctoral Training in Future Power Networks and Smart Grids (EP/L015471/1) with industry support from Rolls-Royce plc.

\section{REFERENCES}

[1] M. Tabari, S. Member, A. Yazdani, and S. Member, "A Mathematical Model for Stability Analysis of a DC Distribution System for Power System Integration of Plug-In Electric Vehicles," vol. 64, no. 5, pp. 1729-1738, 2015

[2] H. Chen, Z. Hu, H. Luo, J. Qin, R. Rajagopal, and H. Zhang, "Design and planning of a multiple-charger multiple-port charging system for pev charging station," IEEE Transactions on Smart Grid, pp. 1-1, 2017. 
[3] W. Tushar, C. Yuen, S. Huang, D. B. Smith, and H. V. Poor, "Cost minimization of charging stations with photovoltaics: An approach with EV classification," IEEE Transactions on Intelligent Transportation Systems, vol. 17, no. 1, pp. 156-169, 2016.

[4] A. Asthana and M. Taylor, "Britain to ban sale of all diesel and petrol cars and vans from 2040," 2017. [Online]. Available: https://www.theguardian.com/politics/2017/jul/25/britain-toban-sale-of-all-diesel-and-petrol-cars-and-vans-from-2040

[5] M. Taylor, "Volvo's Electric Car Strategy Is Being Badly Misrepresented," 2017. [Online]. Available: https://www.forbes.com/sites/michaeltaylor/2017/07/05/mostof-what-youve-read-on-volvos-electric-car-strategy-is-badlymisleading/\#1 cae58a72ed9

[6] C. Roe, F. Evangelos, J. Meisel, A. P. Meliopoulos, and T. Overbye, "Power system level impacts of PHEVs," Proceedings of the 42nd Annual Hawaii International Conference on System Sciences, HICSS, pp. 1-10, 2009.

[7] V. K. Channegowda, Janamejaya Pathipati, "Comprehensive Review and Comparison of DC Fast Charging Converter Topologies : Improving Electric Vehicle Plug-to-Wheels Efficiency," pp. 263-268, 2015.

[8] J. Y. Yong, V. K. Ramachandaramurthy, K. M. Tan, A. Arulampalam, and J. Selvaraj, "Modeling of electric vehicle fast charging station and impact on network voltage," Clean Energy and Technology (CEAT), 2013 IEEE Conference on, pp. 399-404, 2013.

[9] H. Zhang, W. Tang, Z. Hu, Y. Song, Z. Xu, and L. Wang, "A method for forecasting the spatial and temporal distribution of PEV charging load," IEEE Power and Energy Society General Meeting, no. October, pp. 1-5, 2014.

[10] Y. Guo, S. Member, J. Xiong, S. Member, S. Xu, and S. Member, "TwoStage Economic Operation of Microgrid-Like Electric Vehicle Parking Deck," IEEE Transactions on Smart Grid, vol. 7, no. 3, pp. 1703-1712, 2016.

[11] P. Zhang, K. Qian, C. Zhou, B. G. Stewart, and D. M. Hepburn, "A Methodology for Optimization of Power Systems Demand Due to Electric Vehicle Charging Load," IEEE Transactions on Power Systems, vol. 27, no. 3, pp. 1628-1636, 2012.

[12] A. Gusrialdi, Z. Qu, and M. A. Simaan, "Distributed Scheduling and Cooperative Control for Charging of Electric Vehicles at Highway Service Stations," IEEE Transactions on Intelligent Transportation Systems, pp. $1-15,2017$.

[13] J. Ugirumurera and Z. J. Haas, "Optimal Capacity Sizing for Completely Green Charging Systems for Electric Vehicles," IEEE Transactions on Transportation Electrification, vol. 7782, no. c, pp. 1-13, 2017.

[14] M. G. Vayá and G. Andersson, "Self Scheduling of Plug-In Electric Vehicle Aggregator to Provide Balancing Services for Wind Power," IEEE Transactions on Sustainable Energy, vol. 7, no. 2, pp. 886-899, 2016.

[15] A. S. A. Awad, M. F. S. Awad, T. H. M. El-fouly, S. Member, E. F. El-saadany, S. Member, and M. M. A. Salama, "Optimal Resource Allocation and Charging Prices for Benefit Maximization in Smart PEVParking Lots," vol. 8, no. 3, pp. 906-915, 2017.

[16] J. A. Azzolini and M. Tao, "A Load-Managing Photovoltaic System for Electric Vehicle Charging," pp. 737-741, 2017.

[17] M. Tabari and A. Yazdani, "An Energy Management Strategy for a DC Distribution System for Power System Integration of Plug-In Electric Vehicles," IEEE Transactions on Smart Grid2, vol. 7, no. 2, 2016.

[18] W. Pei, W. Deng, X. Zhang, H. Qu, and K. Sheng, "Potential of Using Multiterminal LVDC to Improve Plug-In Electric Vehicle Integration in an Existing Distribution Network," IEEE Transactions on Industrial Electronics, vol. 62, no. 5, pp. 3101-3111, 2015.

[19] J. C. Hernández, F. S. Sutil, and P. G. Vidal, "Electrical protection in a smart dc node that feeds electric vehicles charging stations," IET Conference Publications, vol. 2014, no. 626 CP, pp. 1-6, 2014.

[20] A. Ghazanfari, M. Hamzeh, and Y. Abdel-Rady I. Mohamed, "A Resilient Distributed Decentralized Control Framework for DC Parking Lots," IEEE Transactions on Smart Grid, vol. 3053, no. c, pp. 1-1, 2016. [Online]. Available: http://ieeexplore.ieee.org/document/7549038/

[21] P. Blyth, "Technical Article Understanding AC / DC power supply efficiency the hot topic,” Tech. Rep., 2010. [Online]. Available: http://xppower.com/pdfs/TA_Understanding ACDC_0810.pdf

[22] H. Zhang, Z. Hu, Z. Xu, and Y. Song, "Optimal Planning of PEV Charging Station With Single Output Multiple Cables Charging Spots," IEEE Transactions on Smart Grid, pp. 1-10, 2016.

[23] C. Jin, J. Tang, and P. Ghosh, "Optimizing electric vehicle charging with energy storage in the electricity market," IEEE Transactions on Smart Grid, vol. 4, no. 1, pp. 311-320, 2013.
[24] IET Standards, "Code of Practice for Electric Vehicle Charging Equipment Installation," Tech. Rep., 2015.

[25] K. A. Smith, S. J. Galloway, A. Emhemed, and G. M. Burt, "Feasibility of Direct Current Street Lighting \& Integrated Electric Vehicle Charging Points," in IET Hybrid Electric Vehicle Conference 2016, pp. 1-6.

[26] J. Foxall, "Electric car drivers face queues and quarrels," 2017. [Online]. Available: https://www.telegraph.co.uk/cars/features/electriccar-drivers-could-face-queues-quarrels-christmas/

[27] BSI Standards Publications, "Electric vehicle conductive charging system, Part 1: General Requirements," 2014

[28] K. Smith, D. Wang, A. Emhemed, S. Galloway, and G. Burt, "Overview Paper on: Low Voltage Direct Current Distribution System Standards," International Journal Power Electronics, vol. 9, no. 3, pp. 287-310, 2018.

[29] M. Tabari and A. Yazdani, "Stability of a dc Distribution System for Power System Integration of Plug-In Hybrid Electric Vehicles," IEEE Transactions on Smart Grid, vol. 5, no. 5, pp. 2564-2573, 2014.

[30] A. Riccobono and E. Santi, "Comprehensive review of stability criteria for DC power distribution systems," IEEE Transactions on Industry Applications, vol. 50, no. 5, pp. 3525-3535, 2014.

[31] J. Voelcker, "World's largest electric-car charging site in parking facility: in Norway, of course," Green Car Reports, 2017.

[32] IBM, "CPLEX Optimizer," 2017. [Online]. Available: https://www-01.ibm.com/software/commerce/optimization/cplexoptimizer/\#1cae58a72ed9

[33] S. Vagropoulos and A. Bakirtzis, "Optimal Bidding Strategy for Electric Vehicle Aggregators in Electricity Markets," IEEE Transactions on Power Systems, vol. 28, no. 4, pp. 4031-4041, 2013.

[34] Idaho National Laboratory (INL), "Plugged In: How Americans Charge Their Electric Vehicles," 2015.

[35] Elexon, "Load Profiles and their use in Electricity Settlement," Tech. Rep. November 2013, 2013. [Online]. Available: http://www.elexon.co.uk/wpcontent/uploads/2013/11/load_profiles_v2.0_cgi.pdf

[36] SSE, "SSE Economy 10 Pricing for G1 1RD." [Online]. Available: https://sse.co.uk

[37] K. Schaps, "Shell launches fast-charging stations for electric vehicles," oct 2018. [Online]. Available: http://www.independent.co.uk/news/business/news/shell-electric-carsfast-charging-stations-vehicles-uk-netherlands-england-a8006206.html

[38] Evolt, "Evolt Mode 4 Compact Rapid Charger Dual 50kW CHAdeMO Lead \& 32amp Type 2 AC Lead.” [Online]. Available: http://www.rexelenergysolutions.co.uk/product/2500802825/EvoltMode-4-Compact-Rapid-Charger-Dual-50kW-CHAdeMO-Lead-\&32amp-Type-2-AC-Lead

[39] Midshire Electrical \& Lighting, "ROLEC EV Basic Charge Double IEC Socket (16A/32A)." [Online]. Available: https://www.midselec.co.uk/rolec-ev-basic-charge-doubleiec-socket-16a-32a.html

[40] Superlec Direct-2, "Superelec 6944X 4-Core 240MM SWA BS5467 Steel Wire Armoured Cable - Harmonised Cores." [Online]. Available: https://www.superlecdirect.com/p-6944x240-superlec-6944x-4core240mm-swa-bs5467-steel-wire-armoured-cable-harmonised-cores/

Lesiba Mokgonyana received his M.Eng. degree in electrical engineering from the University of Pretoria, South Africa, in 2015. From 2010 to 2014, he worked in the power industry as an engineer with the distribution division of Eskom, South Africa. He is currently pursuing the Ph.D. degree in electronic and electrical engineering with the University of Strathclyde, UK. His research interests include planning and operation of power systems with particular focus on optimization of distributed energy resources in distribution systems.

Kyle Smith received his M.Eng. degree in Mechanical Engineering from the University of Edinburgh in 2011 and has previously worked internationally in the field of renewable energy development for both commercial and community owned projects. He is currently a Ph.D. candidate at the University of Strathclyde with a focus on Low Voltage Direct Current distribution networks for electric transportation applications. 
Stuart Galloway received his Bachelors degree in Mathematical Sciences form the University of Paisley in 1992. He obtained his M.Sc. degree in Nonlinear Modelling (1993) and Ph.D. in Numerical Analysis (1998) from the University of Edinburgh, UK. Since 1998 he has been researching optimisation problems in power systems, electricity markets and novel architectures relating to aero and marine systems. He is currently a Professor in the Institute for Energy and Environment at the University of Strathclyde. 